\title{
PNEUMATOSIS INTESTINALIS: SPONTANEOUS CLINICAL AND RADIOLOGICAL RESOLUTION
}

\author{
S. V. Navani, M.B., B.S., F.F.R., D.M.R.D. \\ Senior Registrar, Department of Diagnostic Radiology, \\ Middlesex Hospital and Royal Northern Hospital, London
}

\begin{abstract}
The Aetiology of pneumatosis intestinalis in most cases is either unknown or open to discussion. Advice and treatment is often a problem. The following case is reported as an example of the group in which recovery was spontaneous.

The condition was first described by Du Vernoi in 1738 and much has been written on the subject since then. The various terms that have been used in the past to describe this condition include, intestinal emphysema, gas cysts of intestine, pseudocystic disease of intestine, pneumatosis cystoides intestinalis, cystoides intestinorum hominis and peritoneal pneumatosis.

The condition may occur in any part of the bowel from the stomach to the rectum and may also occur in the mesentery. The collection of air may be linear or more commonly in the shape of cysts. This latter appearance is the explanation for the use of the word 'cystoides' in naming the condition.
\end{abstract}

\section{Case Report}

I.M., a well-nourished Cypriot of Turkish extraction, male, aged 37 years presented on 24th May, 1963, with the chief complaint of passing motions every hour, accompanied by tenesmus.

Investigations Stools: Negative for pathogens and parasites. Hb., $86 \%$, PCV, $43 \%$, MCHC, $30 \%$, WBC, $8000 / \mathrm{cu}$. cm., polys, $64 \%$, eosinos, $3 \%$, lymphs, $31 \%$, monos, $2 \%$.

Sigmoidoscopy: (25.5.'63) Proctocolitis up to $24 \mathrm{~cm}$. Chest Radiographs: Normal. Barium Enema Examination Report: (29.5.'63) (Fig. 1) Barium flows easily up to caecum. The descending colon contains redundant loops. The outline of the colon from the distal part of the transverse colon to the sigmoid colon is irregular and this irregularity is due to indentation of the barium-filled lumen by numerous air cysts in its wall. Conclusions: There is pneumatosis cystoides intestinalis affecting the distal colon.

The patient was treated conservatively and soon became asymptomatic. Sigmoidoscopy was repeated on 28.6.' 63 and showed resolution of procto-colitis. Barium enema examination: on 10.3.'64, normal (Fig. 2).

\section{Discussion}

In a review of the world literature, Koss (1952) was able to find reference to 255 cases and in his cases, the small bowel was affected in $63 \%$ and the large bowel in $22 \%$; of the latter almost four fifths of the lesions were

Present address:

Boston City Hospital, Boston, Mass., U.S.A. in the caecum and the surrounding area. Thus pneumatosis involving the left half of the colon is relatively uncommon, and in the review by Williams (1961) only forty cases of this type could be found in the literature. The condition is more commonly encountered in males, the male:female ratio being $4: 1$. The common age incidence is between $20-50$ years.

The disease can be classified into two types:

1. The primary type not associated with any other lesion.

2. The secondary type associated with:

(a) Obstruction due to duodenal or pyloric ulceration, volvulus or tumour.

(b) Trauma following sigmoidoscopy, biopsy, pneumoperitoneum and pre-sacral air studies.

(c) Secondary to pulmonary disease with severe cougtb Keyting and his associates (1961) believe that mos if not all cases can be explained on the basis of intee stitial emphysema in the lung, reaching the bowel walt via the mediastinum, retroperitoneal tissues and blood vessels. Kenny (1963) makes the point that a disturbance of acid-base balance plays an important role in the aetiology of pneumatosis. However, by general agreement, primary pneumatosis intestinalis still remains a condition of unknown aetiology.

There is no characteristic picture associated with pneumatosis intestinalis and it is often diagnosed in a fortuitous way. (Goligher, 1961). The diagnosis may be made on plain films (Kenny, 1963; Jakimov and Sheehan, 1963) but is more often made during barium enema examination in the investigation of abdominal pain, diarrhoea and/or passage of blood and mucus. Sigmoidoscopy is of help if the cysts are situated in the submucosa when numerous grape-like swellings looking like multiple polyps can be seen. In most cases, these cysts are located subserosally and are therefore seen as a cluster of pellucid cysts at operation or necropsy.

On sigmoidoscopy and barium studies, this condition has teen mistaken for multiple polyposis, polypoidal change in ulcerative colitis or carcinoma. It should, however, be readily recognized on X-ray films. The gas cysts are excessively radiolucent and have wide bases. Correct diagnosis is important as pneumatosis coli is essentially benign and needs by itself no surgical intervention. Surgical treatment should be reserved only for the cases complicated by obstruction, haemorrhage or volvulus. (Williams, 1961). Presumably in the present case there is a relationship between the procto-colitis and the pneumatosis but the nature of this must remain conjectural. In a recent paper by Colquhoun (1965) 


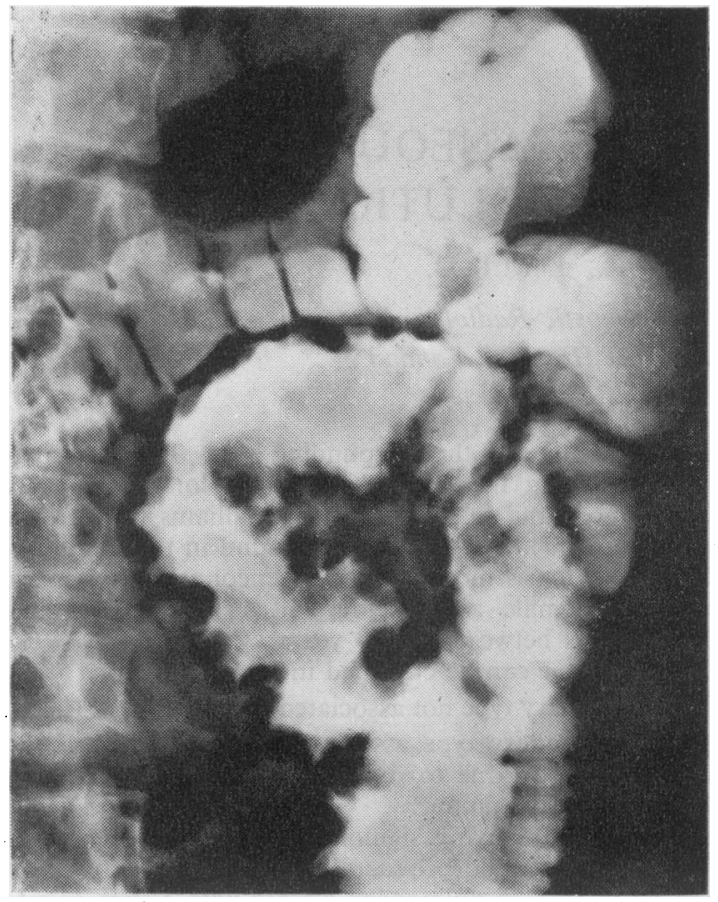

FIG. 1.-Barium enema (29.5.63). Large_air cysts in the wall of the sigmoid colon.

another case where spontaneous regression occurred within 15 months is recorded.

\section{Summary}

A case of pneumatosis affecting the distal colon is described.

The condition is briefly discussed.

Spontaneous resolution, clinically and radiologically occurred.

I am grateful to Dr. L. S. Carstairs for his assistance and advice in the preparation of this paper.

I am also indebted to Mr. D. L. B. Farley, F.R.C.S., for permission to publish the details of this patient who was under his care.

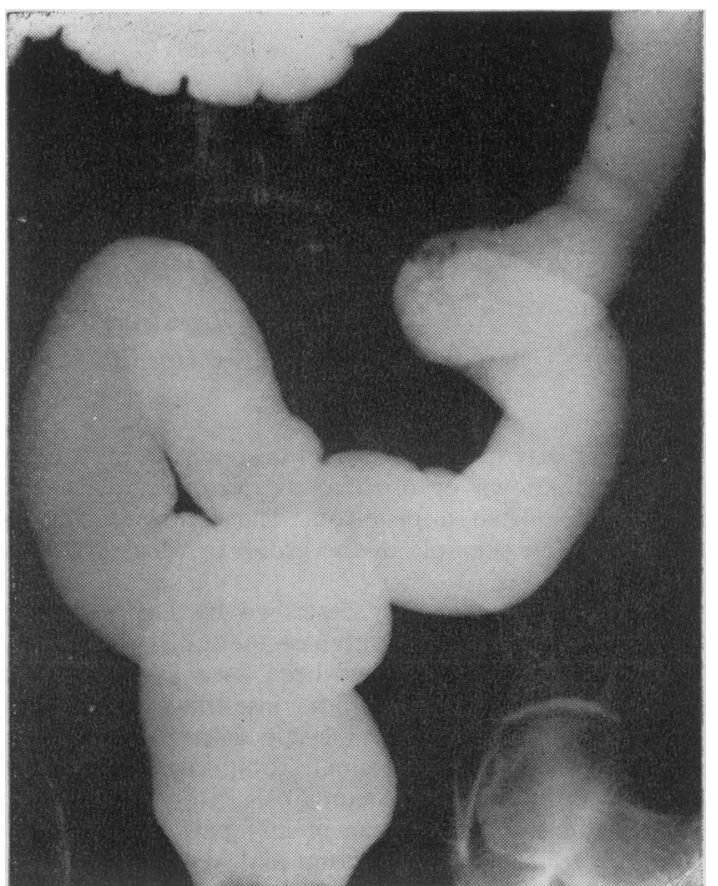

FIG. 2.-Barium enema (10.3.64). The appearances are now normal.

\section{REFERENCES}

Colquhoun, J. (1965): Intramural Gas in Hollow Viscera, Clin. Radiol., 16, 71 .

Golighter, J. G. (1961): Surgery of the Anus, Rectum and Colon. London: Cassell.

JAKIMOV, I. L., and SheehaN, F. R. (1963): Case Report: Pneumatosis Intestinali, Brit. J. Radiol., 36, 695.

Kenny, J. C. (1963): Pneumatosis Intestinalis, Clin. Radiol., 14, 70.

KeYting, W. S., McCarver, R. R., Kovarik, J. L., and DAYwIT, A. L. (1961): Pneumatosis Intestinalis: A New Concept, Radiology, 76, 733.

Koss, L. G. (1952): Abdomonal Gas Cysts (Pneumatosis Cystoides Intestinorum Hominis). An Analysis with a Report of a Case and Critical Review of the Literature, A.M.A. Arch. Path., 53, 523.

Williams, J. L. (1961): Pneumatosis Cystoides Intestinalis involving the Left Half of the Colon and Rectum, Brit. J. Surg., 49, 213, 67. 